\title{
Efeitos Fisiológicos Agudos da Taurina \\ Contida em uma Bebida Energética em \\ Indivíduos Fisicamente Ativos
}

\section{Acute Physiological Effects of Taurine Content of an Energy Drink in Physically Active Subjects}

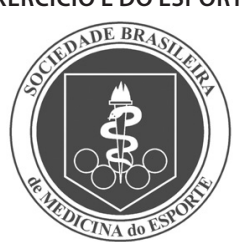

Artigo Original
Tatyana Dall'Agnol',

Paulo Fernando Araújo de Souza ${ }^{2}$

1. Mestre em Educação Física pela Universidade Católica de Brasília na área de concentração de Saúde e Atividade Física.

2. Doutor em Bioquímica pela Universidade de Oxford e Diretor do Curso de Nutrição da Universidade Católica de Brasília.

\section{Endereço para correspondência:}

Tatyana Dall'Agnol

SQS 105, Bloco B, apto. 204

- 70344-020 - Brasília-DF.

E-mail: dagnol@terra.com.br

Submetido em 24/05/2006

Versão final recebida em 04/09/2007

Aceito em 24/10/2008

\section{RESUMO}

Segundo a Secretaria de Vigilância Sanitária do Ministério da Saúde, bebidas energéticas são identificadas como compostos líquidos prontos para o consumo, sendo estas constituídas de carboidratos, taurina, cafeína, glucoronolactona, inositol e vitaminas do complexo B. Existem poucas pesquisas sobre o uso de taurina contida em bebidas energéticas relacionado com a melhora de desempenho. Este trabaIho teve como objetivo analisar as respostas metabólicas e hemodinâmicas decorrentes da administração da associação de taurina e cafeína durante teste ergoespirométrico em indivíduos fisicamente ativos. Para esse fim, 20 indivíduos do sexo masculino, $26 \pm 4,32$ anos e índice de massa corporal 23,79 \pm 2,95, praticantes de atividades aeróbicas, foram submetidos a duas sessões de testes em cicloergômetro ligado a analisador metabólico de gases. $O$ esquema das sessões foi duplo- cego e 60 minutos antes do início dos testes foi oferecida bebida experimental ou bebida placebo. Durante os testes, foram mensuradas: frequência cardíaca (FC), pressão arterial sistólica (PAS) e diastólica (PAD), lactato sanguíneo (Lac), percepção subjetiva de esforço por escala de Borg (PSE), consumo máximo de oxigênio $\left(\mathrm{VO}_{2 \text { máx }}\right)$, consumo de oxigênio no ponto de compensação respiratório (RCP), tempo de exercício (TE) e carga de trabalho (CAR). Para a análise dos dados, foi realizado um teste t pareado $(p \leq 0,05)$. Na carga de trabalho, os resultados indicaram que houve aumento de 10 watts com a administração da bebida experimental, contudo, sem significância estatística (BE: $342 \pm 40,60 ;$ P: $332,50 \pm 56,83$ ). Os principais resultados deste estudo indicam que a administração de taurina contida em bebida energética não influenciou os resultados das variáveis investigadas. Assim, podemos concluir que a dose de $2 \mathrm{~g}$ utilizada não foi capaz de aumentar o desempenho.

Palavras-chave: desempenho, suplementação, ergogênico.

\begin{abstract}
According to the Sanitary Surveillance Agency of the Ministry of Health, energy drinks are identified as liquid compounds ready for consumption, being made of carbohydrates, taurine, caffeine, glucoronolactone, inositol, and B-complex vitamins. Given the small number of studies on the use of taurine in energy drinks related to improved performance, this paper aimed to analyze the metabolic and haemodynamic responses resulted from the administration of the association of taurine and caffeine during an ergospyrometric test in physically active subjects. Therefore, twenty male individuals, $26 \pm 4.32$ years and body mass $23.79 \pm 2.95$, frequent practitioners of aerobic activities, were submitted to two test sessions in cycle ergometer hooked to a gas metabolic analyzer. The sessions schedule was double-blind, and 60 minutes before them the individuals were offered experimental drinks or placebo drinks. During the tests, the subjects were evaluated on the following variables: heart rate (HR), systolic blood pressure (SBP), diastolic blood pressure (DBP), blood lactate (Lac), subjective perceived exertion by Borg scale (SPE), maximum oxygen uptake $\left(\mathrm{VO}_{2 \text { max }}\right)$, oxygen uptake at the compensation respiratory point (CRP), exercise time (ET) and work load (WL). A paired test was carried out for data analysis, where $(p \leq 0.05)$. On the work load, the results indicated an increase of 10 watts with the administration of the experimental drink, with no statistical significance, though. (ED: 342 $\pm 40.60 ; \mathrm{P}: 332.50 \pm 56.83$ ). The main results of this study point out that taurine administration contained in the energy drink did not influence in the levels of the investigated variables. Thus, we can conclude that the $2 \mathrm{~g}$ dose used did not improve performance.
\end{abstract}

Keywords: performance, supplementation, ergogenic 


\section{INTRODUÇÃO}

A Secretaria de Vigilância Sanitária do Ministério da Saúde publicou, no Diário Oficial da União de 5 de novembro de 1998, a Portaria n 868, regulamentando a produção e a venda de bebidas energéticas, identificando-as como compostos líquidos prontos para o consumo. Analisando a composição dessas bebidas, observa-se que a grande maioria consiste numa mistura de carboidratos (cerca de $11 \mathrm{~g} / \mathrm{dl}$ ), taurina (cerca de $400 \mathrm{mg} / \mathrm{dl}$ ), cafeína (cerca de $32 \mathrm{mg} / \mathrm{dl}$ ), glucoronolactona (cerca de 240mg/dl), inositol (cerca de 20mg/dl) e vitaminas do complexo B (40\% a 100\% das necessidades diárias).

O interesse pela taurina (Tau), iniciou-se em meados de 1960, após a constatação de sua presença em diversos tecidos corporais. As primeiras publicações sobre essa substância datam da década de 60, com grande aumento do número de trabalhos a partir da década de 70 , os quais, em sua maioria, evidenciavam as possíveis aplicações clínicas e o envolvimento da Tau na prevenção da degeneração da retina, epilepsia e ataxias ${ }^{(1)}$.

A Tau ou ácido beta-aminossulfônico é um composto final do metabolismo dos aminoácidos sulfurados (metionina e cisteína) que se encontra conjugada com ácidos biliares de sódio e potássio, resultando na formação do ácido taurocólico, um dos ácidos da bile alcalina, essencial para absorção das gorduras ${ }^{(2)}$. Está presente em altas concentrações em algas e no reino animal. A síntese de Tau ocorre a partir dos aminoácidos metionina e cisteína, através de uma sequência de reações enzimáticas de oxidação e transulfuração que requerem a participação da vitamina B6 como cofator. Portanto, sem dúvida que menor ingestão desses aminoácidos pode acarretar aumento das necessidades de Tau. As necessidades dos aminoácidos sulfurados em adultos correspondem a $17 \mathrm{mg} / \mathrm{g}$ de proteína ingerida(3-5). A maior concentração de Tau ocorre naturalmente em frutos do mar (8.270mg/kg para crustáceos, $5.200 \mathrm{mg} /$ $\mathrm{kg}$ para moluscos e $6.550 \mathrm{mg} / \mathrm{kg}$ para mexilhões), pescada $(1.720 \mathrm{mg} /$ $\mathrm{kg})$, carne escura de aves $(2.000 \mathrm{mg} / \mathrm{kg}$ para frango e $3.000 \mathrm{mg} / \mathrm{kg}$ para peru) ${ }^{(6)}$. A ingestão diária de Tau exerce papel importante na manutenção do pool desse aminoácido no organismo, uma vez que, nos mamíferos, a habilidade de sintetizá-la é limitada ${ }^{(7)}$.

Estudo realizado com 10 atletas de endurance a 60 minutos de ciclismo submáximo, a $70 \%$ do $\mathrm{VO}_{2 \text { máx' }}$ com subsequente protocolo incremental até a exaustão, demonstrou aumento significante do tempo de exaustão, diminuição significante das catecolaminas e, consequente, melhora de desempenho após a ingestão de $500 \mathrm{ml}$ de bebida energética contendo Tau. Tais efeitos se devem à maior economia cardíaca e ao efeito inotrópico positivo da Tau ${ }^{(8)}$. O importante efeito positivo na resistência se deve às múltiplas ações bioquímicas da Tau que têm sido descritas tanto em circunstâncias saudáveis, como em situações de deficiência cardía$\mathrm{ca}^{(3,9,10)}$. Outros autores ${ }^{(11)}$ também investigaram os efeitos metabólicos e cardíacos relacionados com a Tau em atletas e demonstraram que ela previne o aumento da creatina quiinase (CK) e inibe a diminuição das proteínas totais no soro nas primeiras 24 horas após o exercício. A Tau exerce efeito protetor quando o coração está sob situações de estresse. Esse aminoácido derivado aumenta a função cardíaca através da regulação da homeostasia intracelular de cálcio ${ }^{(10)}$. Esse efeito inotrópico é devido ao aumento da taxa de bombeamento e da quantidade de cálcio nas proteínas miofibrilares contráteis. Essas ações farmacológicas e fisiológicas da Tau são muito similares às das características dos digitálicos ${ }^{(8)}$.

Após investigação ecocardiográfica, antes e depois do exercício, foi relatado aumento na contratilidade do átrio esquerdo, maior fração de encurtamento cardíaco (fractional shortening), acompanhada de grande ejeção sanguínea após o consumo de Tau ${ }^{(12)}$. O mecanismo envolvido pode ser explicado pelo efeito inotrópico positivo da Tau ${ }^{(8,11)}$.

Observa-se na literatura científica grande carência de estudos relacionados à ingestão de taurina contida em uma bebida energética no que diz respeito à melhora de desempenho. Além disso, relatos de praticantes de atividades físicas e atletas, usuários dessas bebidas, sobre seus efeitos positivos no desempenho, têm levado ao consumo das mesmas no esporte. Entretanto, ainda existem poucas evidências científicas se as bebidas energéticas de fato possuem algum efeito ergogênico. O presente estudo teve como objetivo analisar as respostas metabólicas e hemodinâmicas decorrentes da administração de taurina contida em uma bebida energética durante teste ergoespirométrico em indivíduos fisicamente ativos.

\section{METODOLOGIA}

Foram avaliados 22 indivíduos do sexo masculino, aparentemente saudáveis (aptos a partir de investigação através de questionário para patologia infecciosa ou crônico-degenerativa), não-fumantes, com idade entre 20 e 36 anos, sendo todos praticantes de atividade física regular de, no mínimo, três vezes por semana, modalidades aeróbicas com tempo de treinamento havia no mínimo um ano. Os voluntários foram selecionados no Curso de Educação Física da Universidade Católica de Brasília.

Foram excluídos indivíduos não saudáveis, praticantes de atividade física com frequência inferior a três vezes por semana, somente praticantes de atividades anaeróbias (alta intensidade e curta duração), com tempo de treinamento inferior a um ano, fazendo uso de esteroides anabólicos e aqueles que não completaram todos os testes.

De acordo com os critérios estabelecidos, durante o estudo, os testes de dois participantes foram excluídos por apresentar artefatos (falhas de análise) durante a ergoespirometria, o que dificultou a leitura dos resultados. Dessa forma, a amostra final foi composta de 20 indivíduos praticantes de atividade física.

O estudo foi aprovado pelo comitê de ética da Universidade Católica de Brasília através do registro CEP/UCB 072/2004. Todos os participantes, após ser previamente esclarecidos sobre os objetivos, procedimentos, possíveis desconfortos, riscos e benefícios do estudo, assinarem um termo de consentimento livre e esclarecido.

Análise estatística: foi utilizado teste $t$ de Student pareado, para analisar as respostas metabólicas e hemodinâmicas decorrentes da administração da bebida energética com taurina durante teste ergoespirométrico. O nível de significância para os testes estatísticos foi estabelecido em $5 \%(p \leq 0,05)$.

\section{PROCEDIMENTOS}

Os indivíduos foram submetidos a duas sessões de testes, quando receberam de forma randomizada e duplo-cega, uma solução Experimental ( $B_{1}$ : bebida contendo taurina 2g, cafeína 160mg, glicose 10,5g e sacarose 43g) e, em outro momento do teste, uma solução Placebo ( $\mathrm{B}_{2}$ : bebida contendo cafeína $160 \mathrm{mg}$, glicose $10,5 \mathrm{~g}$ e sacarose $43 \mathrm{~g}$ ). As bebidas $(500 \mathrm{ml})$ foram preparadas em farmácia de manipulação e administradas 60 minutos antes do início do exercício. O intervalo entre as fases foi de sete dias para melhor recuperação. E os avaliados foram orientados a não realizar atividades físicas nos dias anterior e posterior aos testes.

Os voluntários foram submetidos a um protocolo de carga incremental em cicloergômetro Excalibur (Excalibur Lode ${ }^{\circledR}$, Holanda) ligado ao analisador metabólico de gases Cortex Metalyzer (Cortex Biophysik, $\mathrm{DE})$, iniciando com uma carga de 25 watts por um minuto, pedalando à frequência de 60rpm. Sem interrupção, seguia-se o exercício e, a partir do primeiro minuto, foram feitos incrementos sucessivos de 50 watts a cada intervalo de tempo de dois minutos, de modo contínuo, até o esforço máximo ${ }^{(13)}$. Utilizou-se ainda um frequencímetro para registro da frequência cardíaca. Todos os participantes foram encorajados verbalmente ao longo do teste. 
Durante o exercício, o analisador metabólico de gases VO $2000^{\circledR}$, acoplado ao sistema computatorizado Ergopc Elite ${ }^{\circledR}$ versão 2.0 (Micromed(C, Brasília), mediu o volume de ar expirado (VE), o volume de oxigênio consumido por minuto $\left(\mathrm{VO}_{2}\right)$, o volume de dióxido de carbono produzido por minuto $\left(\mathrm{VCO}_{2}\right)$ e a razão de trocas gasosas (RER). Também foram determinadas durante os testes as variáveis frequência cardíaca (FC) utilizando-se Polar Accurex Plus (Interface Polar Plus ${ }^{\circledR} /$ software versão 1.02), pressão arterial sistólica (PAS) e diastólica (PAD) utilizando-se esfigmomanômetro aneroide $\left(\operatorname{Tycos}^{\circledR}\right)$ e estetoscópio $\left(\right.$ Sprague $\left.{ }^{\circledR}\right)$, percepção subjetiva de esforço (Borg) e lactato (Boeringer Mannheim ${ }^{\circledR}$ ).

Imediatamente após, aos quatro e aos nove minutos após o término dos testes experimentais, também foram coletadas amostras de sangue de aproximadamente $20 \mu$ para medir a lactacidemia através do aparelho portátil Accusport (Boeringer Mannheim ${ }^{\circledR}$ ) e fitas para análise BM-Lactate (Roche Diagnostics, Mannheim, Alemanha). As coletas de lactato foram realizadas a partir de punção feita com "caneta"Softclix ${ }^{\circledR}$ nos dedos.

Cada voluntário executou o mesmo protocolo duas vezes (bebida experimental e placebo), sempre utilizando os mesmos critérios.

$\mathrm{Na}$ análise estatística, foi utilizado teste t pareado, para analisar as respostas metabólicas e hemodinâmicas decorrentes da administração da bebida energética com taurina durante teste ergoespirométrico. 0 nível de significância para os testes estatísticos foi estabelecido em $5 \%(p \leq 0,05)$.

\section{RESULTADOS}

A tabela 1 apresenta as informações sobre idade, massa corporal, estatura, IMC e percentual de gordura.

TABELA 1. Característica físicas dos voluntários $(n=20)$

\begin{tabular}{l|c|c|c}
\hline & Média \pm DP & Mínimo & Máximo \\
\hline Idade (anos) & $26 \pm 4,32$ & 20 & 36 \\
\hline Massa corporal $(\mathrm{kg})$ & $72,29 \pm 12,03$ & 54,75 & 91,70 \\
\hline Estatura $(\mathrm{m})$ & $174,04 \pm 6,39$ & 1,61 & 1,85 \\
\hline IMC $\left(\mathrm{kg} / \mathrm{m}^{2}\right)$ & $23,79 \pm 2,95$ & 19,69 & 30,36 \\
\hline Percentual de gordura (\%) & $13,18 \pm 5,30$ & 6,93 & 23,87 \\
\hline
\end{tabular}

Os dados apresentados a seguir representam a média e o desvio padrão. A frequência cardíaca (FC) foi registrada em batimentos/minuto (bpm) e a pressão arterial sistólica (PAS) e diastólica (PAD) em mmHg, a cada dois minutos, durante a execução do protocolo, não havendo diferença estatisticamente significante. Frequência cardíaca ( $B_{1}: 141,55$ $\left.\pm 8,09 ; B_{2}: 139,55 \pm 7,58\right)$, pressão arterial sistólica $\left(B_{1}: 168,90 \pm 15,79\right.$; $\left.B_{2}: 169,55 \pm 21,55\right)$.

Nenhuma diferença estatística foi observada em relação à percepção subjetiva de esforço (Borg) e às concentrações de lactato ( $\mathrm{mmol} / \mathrm{L}$ ) determinadas imediatamente, aos quatro e aos nove minutos após a execução dos testes. Sendo para Borg: $B_{1}(12,70 \pm 1,26)$ e $B_{2}(12,45 \pm$ 1,19); para lactato imediatamente após: $B_{1}(7,12 \pm 3,27)$ e $B_{2}(5,97 \pm 2,57)$; para lactato aos quatro minutos: $B_{1}(7,86 \pm 2,28)$ e $B_{2}(8,61 \pm 3,15)$; e para lactato aos nove minutos: $B_{1}(7,94 \pm 2,87)$ e $B_{2}(7,69 \pm 2,47)$.

A carga atingida, após a ingestão de bebida experimental, foi um pouco maior ao final do teste em comparação com a bebida placebo; entretanto, nenhuma diferença estatística pôde ser observada em resposta à carga máxima obtida em watts após o protocolo experimental, sendo Placebo $(332,50 \pm 56,83)$ versus Bebida Experimental $(342,50 \pm 40,64)$.

Nenhuma diferença estatística pôde ser observada quanto ao consumo máximo de oxigênio $\left(\mathrm{VO}_{2 \text { máx }}\right)$ após o protocolo de carga incremental, sendo $B_{1}(50,10 \pm 9,95)$ versus $B_{2}(49,16 \pm 11,65)$.
Tabela 2. Valores médios de FC, PAS, Borg e carga máxima

\begin{tabular}{|c|c|c|c|}
\hline Grupos & Variável & Média $\pm \mathrm{DP}$ & $\mathrm{P}$ \\
\hline $\mathrm{B}_{1}$ & \multirow{2}{*}{ PAS } & $168,90 \pm 15,79$ & \multirow{2}{*}{$p=0,84$} \\
\hline $\mathrm{B}_{2}$ & & $169,55 \pm 21,55$ & \\
\hline $\mathrm{B}_{1}$ & \multirow{2}{*}{$\mathrm{FC}$} & $187,75 \pm 10,19$ & \multirow{2}{*}{$p=0,85$} \\
\hline $\mathrm{B}_{2}$ & & $187,40 \pm 8,03$ & \\
\hline $\mathrm{B}_{1}$ & \multirow{2}{*}{ Borg } & $12,70 \pm 1,26$ & \multirow{2}{*}{$p=0,38$} \\
\hline $\mathrm{B}_{2}$ & & $12,45 \pm 1,19$ & \\
\hline $\mathrm{B}_{1}$ & \multirow{2}{*}{ CARGA } & $342,50 \pm 40,64$ & \multirow{2}{*}{$p=0,21$} \\
\hline $\mathrm{B}_{2}$ & & $332,50 \pm 56,83$ & \\
\hline
\end{tabular}

$\mathrm{B}_{1}$ : Bebida Experimental

$\mathrm{B}_{2}$ : Placebo

PAS: pressão arterial sistólica

FC: frequência cardíaca

Borg: percepção subjetiva de esforço

O tempo de exaustão obtido para o protocolo de carga incremental nos diferentes grupos Placebo $(13,14 \pm 2,16)$ versus Bebida Experimental $(13,15 \pm 1,41)$ não apresentou diferença estatística, quando foram verificados.

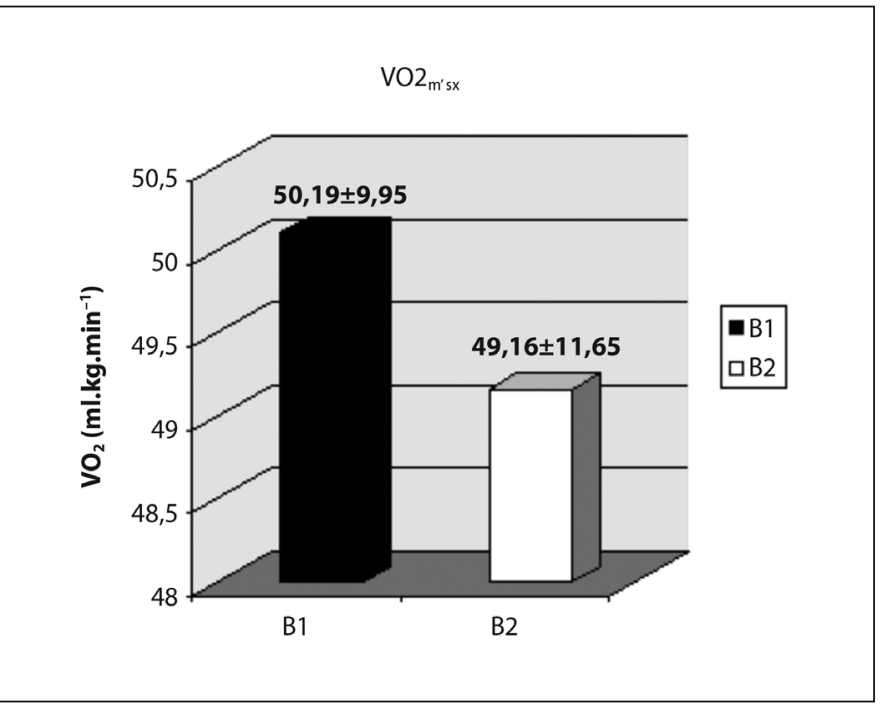

Figura 1. Consumo máximo de oxigênio $\left(\mathrm{ml} / \mathrm{kg} / \mathrm{min}\right.$ ) após a ingestão de $B_{1}$ e $B_{2}$ (média \pm desvio padrão) durante a realização dos testes

\section{DISCUSSÃO}

Um grande número de pesquisas tem sido realizado para identificar substâncias ergogênicas mais efetivas na melhora do desempenho esportivo. Diversos são os trabalhos publicados a respeito dos efeitos dos componentes nas bebidas energéticas, de forma individual, porém, poucas são as informações sobre os efeitos apresentados quando da combinação desses componentes no desempenho.

Os valores da freqüência cardíaca aumentaram gradualmente durante o teste, mas nenhuma diferença estatística significativa foi encontrada. Alguns autores registraram diminuição da FC durante exercícios submáximos após a ingestão de bebida contendo Tau $u^{(8,11,14)}$. Essa redução na FC é induzida pela Tau, que diminui os níveis de norepinefrina no sangue e minimiza a adesão de catecolaminas nas células musculares cardíacas ${ }^{(8)}$.

Estudo realizado em pacientes e em modelos experimentais com altos níveis pressóricos demonstrou que a suplementação de Tau di- 
minuiu os níveis dessa variável, sendo isso decorrente de seus prováveis efeitos nas artérias sanguíneas, no sistema nervoso periférico, nas glândulas ad-renais e nos rins ${ }^{(15-17)}$. Assim, como encontrado em estudo prévio ${ }^{(18)}$, no presente estudo, encontrou-se valor médio de PAS pouco menor para a bebida experimental em comparação com o do placebo, porém, não significativa. Uma diminuição não significativa dessa variável pode ser decorrente da dose de Tau utilizada em nosso estudo (2g), que difere da utilizada nos estudos anteriores (6g).

Não se observou nenhuma diferenciação nas concentrações plasmáticas de lactato devido à presença de suplementação de bebida energética contendo Tau. Entretanto, estudos anteriores ${ }^{(8,19)}$ demonstraram que os níveis de lactato foram significativamente menores no grupo que recebeu tanto bebida energética com Tau ${ }^{(8)}$, como com Tau de forma isolada ${ }^{(19)}$.

Sobre a percepção subjetiva de esforço, os resultados sugerem que o esforço pode ter sido subestimado nos dois testes e que os voluntários não demonstraram noção exata de esforço realizado, uma vez que o teste era máximo. Estudos sugerem que os limites do desempenho no exercício exaustivo podem, em grande extensão, ser psicológicos. Os mecanismos exatos responsáveis por essa fadiga do SNC ainda não são totalmente entendidos, mas o recrutamento do músculo depende, em parte, do controle consciente. O trauma psicológico do exercício exaustivo pode, consciente ou subconscientemente, inibir a disposição do indivíduo. No âmbito da influência psicológica, a motivação aparece com um fator importante que exerce influência na percepção. Somando isso ao fato de que, durante as testagens, foi observada certa ansiedade em relação ao líquido ingerido, que pode atuar como elemento de confusão durante os exercícios e diminuir a percepção, esses fatores (motivação e ansiedade) podem ser entendidos como limitações do estudo(20-22). A literatura científica não é rica nesse sentido, o que dificultou a comparação dessa variável com outros estudos.

O valor médio de $\mathrm{VO}_{2 \text { máx }}$ obtido durante o consumo de $\mathrm{B}_{1}$ não foi significativamente diferente daquele percebido durante o consumo de $\mathrm{B}_{2}$. Os resultados encontrados confirmam achados que demonstraram que, em comparação com um grupo controle, após a ingestão de bebida energética com Tau (3,75 ml/kg), não houve diferença significativa no $\mathrm{VO}_{2 \text { máx }}$ e no $\mathrm{VO}_{2}$ no limiar anaeróbio(23).

\section{REFERÊNCIAS BIBLIOGRÁFICAS}

1. Barbeau A, Huxtable RJ, editors. Taurine and neurological disorders. New York: Raven Press, 1978.

2. Ganong WF, editor. Fisiologia médica. Rio de Janeiro: Prentice-Hall do Brasil, 1993.

3. Huxtable RJ. Physiological actions of taurine. Physiol Rev. 1992;72(1):101-63. Review.

4. Lajolo FM, Tirapegui J, editors. Ciências nutricionais. Rio de Janeiro: Sarvier, 1998.

5. Newsholme EA, Leech AR. Biochemistry for the medical sciences. Acta Physiol Scand. 1983;128:93-7.

6. Laidlaw SA, Grosvenor M, Kopple JD. The taurine content of common foodstuffs. JPEN J Parenter Enteral Nutr. 1990;14(4):183-8.

7. Stapleton PP, O'Flaherty L, redmond HP, Bouchier-Hayes DJ. Host defense - a role for the amino acid taurine? JPEN J Parenter Enteral Nutr. 1998;22(1):42-8. Review.

8. Geib KR, Jester I, Falke M, Hamm M, Waag KL. The effect of a taurine-containing drink on performance in 10 endurance athletes. Amino Acids. 1994;7:45-6.

9. Satoh $\mathrm{H}$, Sperelakis N. Review of some actions of taurine on ion channels of cardiac muscle cells and others. Gen Pharmacol. 1998;30(4):451-63. Review.

10. Azuma J, Sawamura A, Awata N. Uselfulness of taurine in chronic congestive heart failure and its prospective application. Jpn Circ J. 1992;56(1):95-9.

11. Ono M, Watanabe M, Minato, K. Effects of taurine on the metabolism under physical exercise. Sulfur Amino Acids. 1987;10:183-6.

12. Baum M, Weiss M. The influence of at taurine containing drink on cardiac parameters before and after exercise measured by echocardiography. Amino Acids. 2001;20(1):75-82.

13. Balke B, Ware RW. An experimental study of physical fitness of air force personnel. U S Armed Forces Med J. 1959;10(6):675-88.

14. Jester I, Grigereit M, Bernhardt M, Heil S, Banzer W. Effects of ingesting a taurine-enriched, caffeine containg drink on performance and haemodynamics in acyclic trained athletes. Amino Acids. 1997;7:45-6.
Outra característica que não sofreu alteração foi o tempo máximo de execução do protocolo nos diferentes tratamentos. Alguns autores demonstraram aumentos significativos tanto na resistência aeróbica em 9\%, como na resistência anaeróbica em 24\%, após a oferta de bebida energética com Tau em comparação com a bebida controle ${ }^{(18)}$. Tais achados corroboram os resultados de trabalhos anteriores, que também observaram aumento na resistência após a ingestão dessas bebidas $^{(8,14)}$. Esses resultados podem ser provenientes do efeito benéfico da Tau no que diz respeito à função cardíaca, através da regulação na homeostasia de cálcio intracelular pela modulação no fluxo de cátions e pela melhor disponibilidade de cálcio para contração(24,25). Foi observado que a contratilidade cardíaca, especialmente do átrio esquerdo, após o exercício, estava aumentada depois que uma bebida energética com Tau foi oferecida para atletas treinados ${ }^{(12)}$.

A carga máxima produzida em cicloergômetro foi avaliada nos diferentes momentos e a carga atingida após a ingestão de $B_{1}$ foi um pouco maior ao final do teste em comparação com $B_{2}$. Entretanto, essa diferença não foi significativa. Devido à limitação da literatura científica quanto à variável carga de trabalho e ingestão de bebidas energéticas com Tau, não foi possível a comparação dos achados deste estudo.

Apesar de poucos estudos prévios sugerirem que a administração oral de Tau contida em uma bebida energética poderia oferecer benefícios, tais como melhoria no desempenho, os resultados encontrados sugerem que estudos com outras doses, outros indivíduos e modalidades esportivas diferentes sejam realizados para que se verifiquem os benefícios propostos.

Finalmente, este estudo objetivou avaliar as respostas metabólicas e hemodinâmicas decorrentes da administração de Tau contida em uma bebida energética durante teste ergoespirométrico em indivíduos fisicamente ativos. Entretanto, mediante os resultados apresentados, podemos concluir que a suplementação de taurina na dose de $2 \mathrm{~g}$, contida em uma bebida energética no volume de $500 \mathrm{ml}$, não foi capaz de aumentar o desempenho para esse perfil de indivíduos.

Todos os autores declararam não haver qualquer potencial conflito de interesses referente a este artigo.

15. Fujita $T$, Ando $K$, Noda $H$, Ito $Y$, Sato $Y$. Effects of increased adrenomedullary activity and taurine in young patients with borderline hypertension. Circulation. 1987;75(3):525-32.

16. Yamori Y, Nara Y, Ikeda K, Mizushima S. Is taurine a preventive nutritional factor of cardiovascular diseases or just a biological marker of nutrition? Adv Exp Med Biol. 1996;403:623-9. Review.

17. Li N, Sawamura M, Nara Y, Ikeda K, Yamori Y. Direct inhibitory effects of taurine on norepinephrineinduced contraction in mesenteric artery of stroke-prone spontaneously hypertensive rats. Adv Exp Med Biol. 1996;403:257-62.

18. Alford C, Cox H, Wescott R. The effects of Red Bull Energy Drink on human performance and mood. Amino Acids. 2001;21(2):139-50.

19. Manabe S, Kurroda I, Okada K, Morishima M, Okmoto M, Harada N, et al. Decreased blood levels of lactic acid and urinary excretion of 3-methylhistidine after exercise by chronic taurine treatment in rats. J Nutr Sci Vitaminol (Tokyo). 2003;49(6):375-80

20. Borg G, editor. Escala de Borg para dor e o esforço percebido. São Paulo: Manole, 2000.

21. Osse CMC, Cavasini, MS, Matsudo VR. Determinação da sensação subjetiva de esforço em diferentes grupos de idade e de ambos os sexos. Rev Bras Ciências Esporte. 1982;4:17-20.

22. Moura JAR, Peripolli J, Zinn JL. Comportamento da percepção subjetiva de esforço em função da força dinâmica submáxima em exercícios resistidos com pesos. Rev Bras Fisiol Exerc. 2003;2:110-22.

23. Ferreira SE, de Mello MT, Pompéia S, de Souza-Formigoni ML. Effects of energy drink ingestion on alcohol intoxication. Alcohol Clin Exp Res. 2006;30(4):598-605

24. Huxtable R, Bressler R. Effect of taurine on a muscle intracellular membrane. Biochim Biophys Acta. 1973;323(4):573-83.

25. Pasantes-Morales H. Taurine-calcium interactions in frog rod outer segments: taurine effects on an ATP-dependent calcium translocation process. Vision Res. 1982;22(12):1487-93. 